\title{
Comparison of the utility and applicability of the Sokal, Hasford, and EUTOS scores in a population of Chinese patients with chronic-phase chronic myeloid leukemia undergoing imatinib therapy
}

This article was published in the following Dove Press journal: OncoTargets and Therapy

7 September 2015

Number of times this article has been viewed

Leiming $\mathrm{Xia}^{1,2, *}$

Wei Qian ${ }^{3, *}$

Mingzhen Yang ${ }^{3,4}$

Qingsheng $\mathrm{Li}^{4}$

Fei Liu $^{5}$

Yanyan $\mathrm{Xie}^{4}$

'Department of Hematology, The Third Affiliated Hospital of Anhui Medical University, Hefei, People's Republic of China; ${ }^{2}$ Department of Hematology, Hefei Binhu Hospital, Hefei, People's Republic of China; ${ }^{3}$ Department of Hematology, The Fourth Affiliated Hospital of Anhui Medical University, Hefei, People's Republic of China; ${ }^{4}$ Department of Hematology, The First Affiliated Hospital of Anhui Medical University, Hefei, People's Republic of China; ${ }^{5}$ Department of Hematology, The Second People's Hospital of Wuhu, Wuhu, People's Republic of China

*These authors contributed equally to this work
Correspondence: Mingzhen Yang Department of Hematology, The First Affiliated Hospital of Anhui Medical University, Hefei 230022, Anhui,

People's Republic of China

Tel +86 55I 6292204 I

Fax +86 55I 62923655

Email yangmz89@I63.com
Background: Tyrosine kinase inhibitors are increasingly used to treat chronic myeloid leukemia (CML), but loss of complete cytogenetic response (CCyR) indicates treatment failure.

Aim: To compare the efficacy of Sokal, European Treatment Outcome Study (EUTOS), and Hasford prognostic scores with 3-month and 12-month CCyR, event-free survival (EFS) and progression-free survival (PFS) in patients with chronic-phase CML (CP-CML) undergoing imatinib therapy.

Methods: We retrospectively analyzed the outcome of 210 patients with CP-CML treated at the First Affiliated Hospital of Anhui Medical University treated between January 2006 and December 2013. Sokal, EUTOS, and Hasford scores were compared with 3-month and 12-month CCyR, EFS, and PFS.

Results: Kaplan-Meier analyses revealed that 3-month and 12-month CCyR and PFS were lower in patients with high EUTOS scores, and intermediate or high Sokal and Hasford scores (all $P<0.05$ ). Furthermore, EFS was lower in patients with intermediate or high Sokal and Hasford scores (both $P<0.05$ ). Hasford score (hazard ratio $=2.608,95 \%$ confidence interval: 1.473-4.617, $P=0.001$ ) was independently associated with 3-month CCyR.

Conclusion: Although all three scoring systems were associated with EFS, PFS, and 3-month and 12-month CCyR in the Kaplan-Meier analyses (except EFS with EUTOS), only the Hasford score was independently associated with 3m-CCyR, while EUTOS score and Sokal score were not independently associated with any of these outcomes.

Keywords: chronic-phase chronic myeloid leukemia, prognosis, Sokal, EUTOS, Hasford, complete cytogenic response

\section{Introduction}

The etiology of chronic myeloid leukemia (CML) is unknown, but has been attributed to tyrosine kinase activity and genetic translocation of the Philadelphia $(\mathrm{Ph})$ chromosome that carries a fusion gene responsible for the disease. ${ }^{1-6} \mathrm{CML}$ may present in chronic-phase (CP-CML), accelerated-phase, or blast-phase; however, $>90 \%$ of patients are diagnosed in CP-CML. ${ }^{1}$ Over half of all CP-CML cases are asymptomatic $;{ }^{1}$ however, if untreated, these patients will inevitably progress to blast-phase CML within 3-5 years. ${ }^{2}$

Tyrosine kinase inhibitors (TKIs) have greatly improved the prognosis of CML, and projected survival of imatinib-responsive CML is close to $100 \%$ after $6-7$ years. ${ }^{5}$ The IRIS trial achieved 94.9\% 12-month event-free survival (EFS) with imatinib, in comparison to $75.3 \%$ EFS in the control arm. ${ }^{6}$ In approximately $75 \%$ of patients, the $\mathrm{Ph}$ chromosome cannot be detected after 2 years of therapy, a state termed complete 
cytogenetic response (CCyR). CCyR is the main goal of this therapy, and the majority of patients with CML can be maintained in CP-CML..$^{7-9}$

Prognostic scoring systems have been developed for risk stratification of patients with CML. Presently, three prognostic systems are widely accepted in clinical practice: Sokal, ${ }^{10}$ Hasford,,${ }^{11}$ and European Treatment Outcome Study (EUTOS). ${ }^{12}$ The Sokal score is based on patient age and clinical characteristics including spleen size, platelet count, and the percentage of blasts in the peripheral blood, ${ }^{10}$ and the Hasford model also includes eosinophil and basophil counts. ${ }^{11}$ Both systems categorize patients as high, medium, or low risk, but the capacity of such measures to accurately predict response to treatment or inform individualized treatment regimens for CML remains controversial. The Sokal and Hasford systems were designed before the introduction of TKIs, while the EUTOS was created after doubts were raised about the utility of the Sokal and Hasford systems in the TKI era. The EUTOS score is defined only by basophil count and spleen size; a EUTOS score exceeding 87 indicates high risk, and a score of 87 or less indicates low risk. As the EUTOS system involves a lower number of variables, it is easier to use and is reported to accurately predict disease-free survival of CML patients after TKI treatment. ${ }^{13-16}$

The EUTOS score has been used to accurately identify patients with lower probabilities of CCyR and lower survival. ${ }^{12}$ Overall survival and CCyR were also reported to be better predicted by the EUTOS score than the Hasford and Sokal scores in patients receiving imatinib treatment. ${ }^{16}$ A recent study also showed that EUTOS could predict the prognosis in CP-CML patients, but that Sokal and Hasford scores lacked sensitivity. ${ }^{17}$

However, two single-center studies in the US and UK have recently reported that the EUTOS score was not predictive of outcome in patients undergoing TKI treatment. ${ }^{18,19}$ Jabbour et al reported that EUTOS score was not predictive of outcome in 71 patients with early CP-CML treated with standard-dose imatinib. There was no difference between the EFS, major molecular response, or survival of low- and highrisk EUROS groups. Patients undergoing second-generation TKI treatment and with low EUTOS scores did have higher rates of CCyR in comparison to patients with high EUTOS scores, but there was no difference in patients undergoing imatinib treatment. ${ }^{18}$ Marin et al reported that EUTOS score failed to significantly predict progression-free survival (PFS), CCyR, or major molecular response in $282 \mathrm{CP}-\mathrm{CML}$ patients receiving imatinib as first-line therapy. ${ }^{19}$ In addition, the Swedish CML Registry recently reported that Sokal, but not EUTOS, predicted survival in a population-based cohort. ${ }^{20}$
The present study was carried out by analyzing data collected between 2006 and 2013. Guidelines that were applied during this period recommended that cytogenetic analysis be performed at 3-month to 6-month intervals after treatment initiation, and these were the data that were available to the present study. Three-month CCyR was selected because recent studies underlined the importance of achieving early CCyR, ${ }^{21,22}$ the same for 12 months. EFS and PFS were reported and censored on December 1, 2013. No death was observed by this time, preventing the determination of overall survival. In addition, EFS and PFS were preferred to DFS because they provide a more precise appraisal of the evolution of the patients. Thus, the aim of the present study was to compare the capacity of Sokal, Hasford, and EUTOS scores to predict outcome in Chinese patients with CP-CML undergoing TKI treatment. These results could help to refine TKI treatment to specific patients.

\section{Materials and methods Patients}

This was a retrospective study performed in patients diagnosed with CP-CML at the First Affiliated Hospital of Anhui Medical University (Hefei, People's Republic of China) between January 2006 and December 2013. Medical charts were reviewed, and two independent chart reviewers performed data extraction and their results were compared to reduce bias.

Inclusion criteria included: 1) CP-CML diagnosis according to the revised 2011 China CML Diagnostic Criteria, ${ }^{17}$ National Comprehensive Cancer Network, ${ }^{23}$ and European Leukemia Net (ELN) guidelines, ${ }^{21}$ including at least one positive result for the $\mathrm{Ph}$ chromosome and/or BCR-ABL fusion protein, and CML-compatible myelogram and blood cell examination ${ }^{2}$; and 2) patients must have undergone oral imatinib treatment (Novartis, Bale, Switzerland) $400 \mathrm{mg} /$ day. Patients treated with hydroxyurea for $<35$ days were included, but no other treatment was allowed concomitantly with imatinib.

\section{Ethics statement}

The study was approved by the First Affiliated Hospital of Anhui medical University, People's Republic of China. All patients' data were handled and deidentified according to ethical and legal standards. We obtained written informed consent from all participants involved in our study.

\section{Prognosis}

Sokal and Hasford scores were calculated using an online tool (http://www.leukemia-net.org/content/leukemias/ $\mathrm{cml} / \mathrm{cml}$ score/index eng.html). EUTOS scores were also 
calculated using an online tool (http://www.leukemia-net.org/ content/leukemias/cml/eutos score/index eng.html). The

Sokal score is based on age, spleen size, platelet count before treatment, and proportion of blasts in the peripheral blood. Scores are stratified according to low risk $(<0.8)$, intermediate risk (0.8-1.2) and high risk $(>1.2) .{ }^{10}$ The Hasford score is based on age, spleen size, blasts percentage, eosinophil percentage, basophil percentage, and platelet count. Scores are stratified according to low risk $(<780)$, intermediate risk $(781-1,480)$ and high risk $(>1,480) .{ }^{11}$ The EUTOS score is based on the size of the spleen and on the percentage of basophiles in peripheral blood. A EUTOS score $>87$ is stratified as high risk, and $\leq \leq 87$ is low risk. ${ }^{12}$

\section{Follow-up}

Patients' follow-up was examined to determine EFS and PFS. An event was defined as: 1) progression to accelerated or blastphase while taking imatinib; 2) death from any cause while taking imatinib; 3 ) loss of complete hematologic response; or 4) loss of CCyR. ${ }^{22}$ Progression was defined as: 1) progression to accelerated or blast-phase CML while taking imatinib or 2) death from any cause while taking imatinib; 3) loss of complete hematologic response; 4) loss of CCyR; or 5) white blood cell count increased to $>20 \times 10^{9} / \mathrm{L}$ while taking imatinib. ${ }^{22}$

CCyR was defined as the absence of the $\mathrm{Ph}$ chromosome by G-banding analysis of bone marrow, and by $\mathrm{Ph}$ chromosome fluorescence in situ hybridization of peripheral blood cells. ${ }^{24}$ The final observation time for all patients was December 1, 2013.

\section{Outcomes}

The primary outcomes were 3-month and 12-month CCyR. The secondary outcomes were EFS and PFS. In the present study, the Sokal, Hasford, and EUTOS scoring systems were compared with the 3-month and 12-month CCyR, EFS, and PFS, respectively.

\section{Statistical analysis}

Statistical analysis was performed using SPSS 17.0 (IBM, Armonk, NY, USA). If normally distributed, data are presented as means \pm standard deviations; where nonnormally distributed, data are expressed as median (min, max). Categorical variables are expressed as proportions. The KaplanMeier method and log-rank tests were used for the analysis of cumulative incidence of 3-month CCyR (3m-CCyR), 12-month CCyR (12m-CCyR), EFS, and PFS. The Cox regression analysis was used for univariate and multivariate analyses for factors associated with survival using the forward selection method to determine hazard ratios (HRs) and $95 \%$ confidence intervals (95\% CIs). A $P$-value $<0.05$ was considered statistically significant.

\section{Results}

\section{Characteristics of the CP-CML patients}

Table 1 presents the characteristics of the patients at diagnosis. Median age was 42 (range, 6-84) years, and 60\% were male.

\section{Sokal, Hasford, and EUTOS scores}

According to the Sokal score, 28 (13.3\%) patients were high risk, $99(47.1 \%)$ were intermediate risk, and 83 (39.5\%) were low risk. According to the Hasford score, 17 (8.1\%) patients were high risk, $92(43.8 \%)$ were intermediate risk, and 101 (48.1\%) were low risk. According to the EUTOS score, 42 $(20.0 \%)$ patients were high risk and $168(80.0 \%)$ were low risk (Table 1).

\section{Kaplan-Meier analysis}

Kaplan-Meier analyses were performed to assess the cumulative 3m-CCyR and 12m-CCyR in each EUTOS, Sokal, and Hasford score category. The cumulative $3 \mathrm{~m}-\mathrm{CCyR}$ was lower in patients with high EUTOS scores (low vs high, $P<0.001$ ), with intermediate or high Sokal scores (low vs intermediate vs high, $P<0.001$ ), and with intermediate or high Hasford scores (low vs intermediate vs high, $P<0.001$ ) (Figure 1A). The cumulative $12 \mathrm{~m}-\mathrm{CCyR}$ was lower in patients with high EUTOS scores (low vs high, $P<0.001$ ), with intermediate or

Table I Demographic and clinical characteristics of 210 patients with chronic-phase chronic myeloid leukemia at diagnosis

\begin{tabular}{|c|c|}
\hline Characteristics & $\mathbf{N}=\mathbf{2} 10$ \\
\hline Gender (male/female) & $126 / 84$ \\
\hline Age (years) & $42.22(6-84)$ \\
\hline Spleen size $(\mathrm{cm})$ & $6.73(0-21)$ \\
\hline Proportion of blasts (\%) & $1.46(0-10)$ \\
\hline Basophils $\left(10^{9} / \mathrm{L}\right)$ & $4.68(0-18.5)$ \\
\hline Eosinophils $\left(10^{9} / \mathrm{L}\right)$ & $3.5 \mid(0-14.5)$ \\
\hline Platelet count $\left(10^{9} / \mathrm{L}\right)$ & 363.06 (68-989) \\
\hline White blood cell count ( $\left.10^{9} / \mathrm{L}\right)$ & $188.69(2.5-7 \mid 4)$ \\
\hline Hemoglobin $(g / L)$ & $96.68(5|-| 72)$ \\
\hline \multicolumn{2}{|c|}{ European Treatment Outcome Study score, n (\%) } \\
\hline Low risk & I $68(80.0)$ \\
\hline High risk & $42(20.0)$ \\
\hline \multicolumn{2}{|l|}{ Sokal score, n (\%) } \\
\hline Low risk & $83(39.5)$ \\
\hline Intermediate risk & $99(47.1)$ \\
\hline High risk & $28(13.3)$ \\
\hline \multicolumn{2}{|l|}{ Hasford score, n (\%) } \\
\hline Low risk & I0I (48.I) \\
\hline Intermediate risk & $92(43.8)$ \\
\hline High risk & $17(8.1)$ \\
\hline
\end{tabular}

Note: Continuous variables are present as median (min-max). 
A
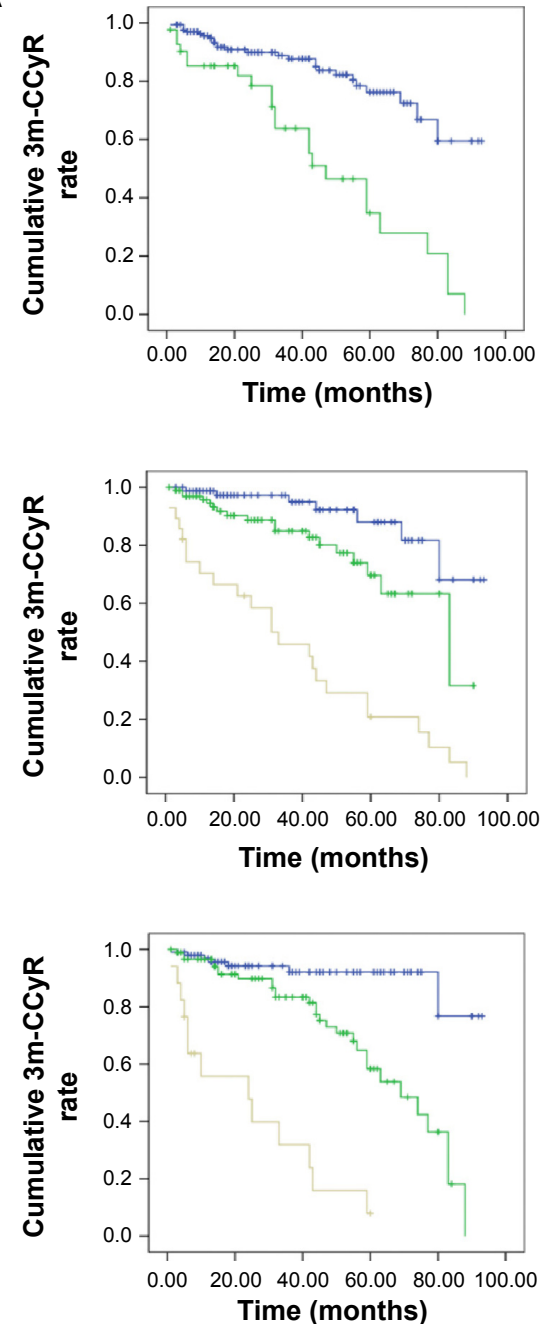

B
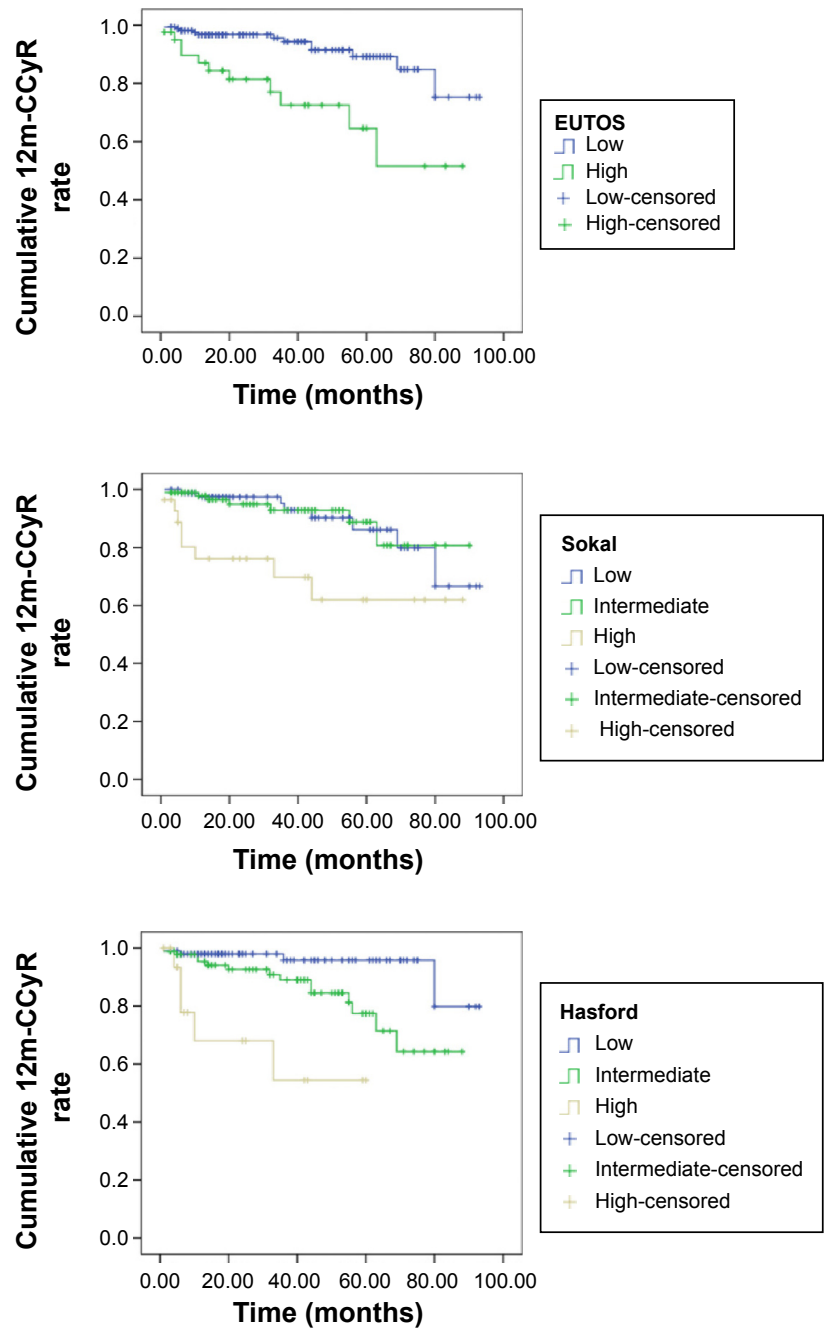

Figure I Kaplan-Meier analysis of cumulative incidence of 3m-CCyR and I2m-CCyR in patients with chronic-phase chronic myeloid leukemia according to EUTOS (low vs high), Sokal (low vs intermediate vs high), and Hasford (low vs intermediate vs high) scores.

Notes: (A) Kaplan-Meier analysis showing the association of $3 \mathrm{~m}-$ CCyR rate with EUTOS (low vs high) scores (log-rank test, $P<0.00 \mathrm{I}$ ), Sokal (low vs intermediate vs high) scores (log-rank test, $P<0.00 \mathrm{I}$ ), and Hasford (low vs intermediate vs high) scores (log-rank test, $P<0.00 \mathrm{I}$ ). (B) Kaplan-Meier analysis showing the association of I $2 \mathrm{~m}$-CCyR rate with EUTOS (low vs high) scores (log-rank test, $P<0.00$ I), Sokal (low vs intermediate vs high) scores (log-rank test, $P=0.004)$, and Hasford (low vs intermediate vs high) scores (log-rank test, $P<0.001$ ).

Abbreviations: CCyR, complete cytogenetic response; EUTOS, European Treatment Outcome Study.

high Sokal scores (low vs intermediate vs high, $P=0.004$ ), and with intermediate or high Hasford scores (low vs intermediate vs high, $P<0.001$ ) (Figure 1B).

Kaplan-Meier analyses were performed using EFS and PFS according to EUTOS, Sokal, and Hasford scores. Events were considered if they happened during the follow-up period. EFS was lower with intermediate or high Sokal scores (low vs intermediate vs high, $P<0.001$ ) and with intermediate or high Hasford scores (low vs intermediate vs high, $P=0.005$ ) (Figure 2A). There was no association between EFS and EUTOS scores $(P=0.289)$. PFS was lower in patients with high EUTOS scores (low vs high, $P=0.011$ ), with intermediate or high Sokal scores (low vs intermediate vs high,
$P=0.007$ ), and with intermediate or high Hasford scores (low vs intermediate vs high, $P<0.001$ ) (Figure $2 \mathrm{~B}$ ).

\section{Multivariate Cox regression analysis}

Multivariate Cox regression analyses were performed. Age (HR=0.949, 95\% CI: 0.919-0.980, $P=0.002)$, proportion of blasts $(\mathrm{HR}=2.178,95 \% \mathrm{CI}: 1.810-2.620, P<0.001)$, and platelet count $(\mathrm{HR}=1.002,95 \% \mathrm{CI}: 1.000-1.004, P=0.045)$ were independently associated with EFS. Age $(\mathrm{HR}=0.909$, 95\% CI: $0.861-0.959, P=0.001)$, proportion of blasts (HR=2.966, 95\% CI: 2.057-4.277, $P<0.001)$, and white blood cell count $(\mathrm{HR}=0.996,95 \% \mathrm{CI}$ : $0.993-0.999, P=0.020)$ were independently associated with PFS. Proportion of blasts $(\mathrm{HR}=1.447,95 \% \mathrm{CI}: 1.262-1.659, P<0.001)$ and 
A
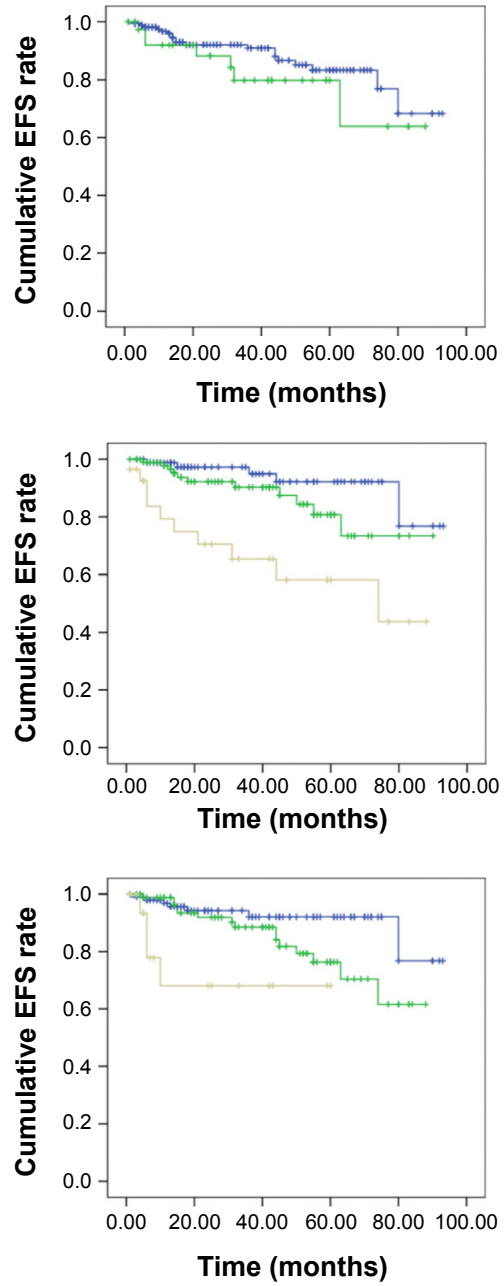

B
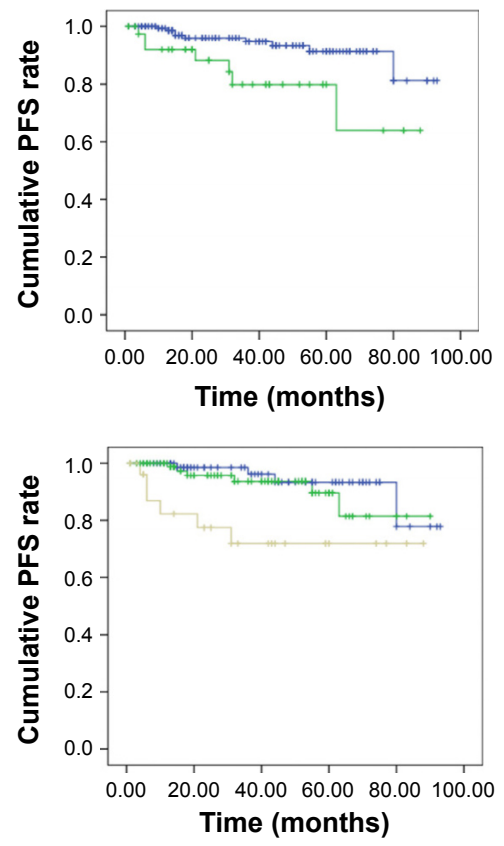

Sokal

$\neg$ Low

$\neg$ Intermediate

High

+ Low-censored

+ Intermediate-censored High-censored

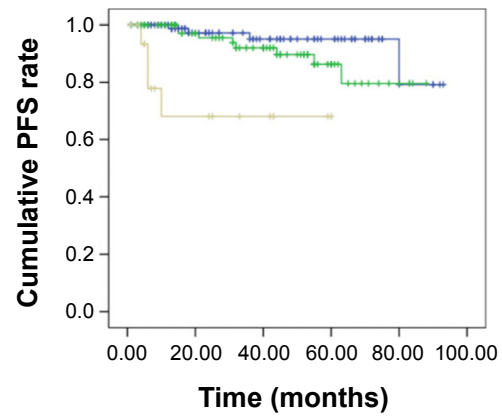

\section{Hasford}

$\neg$ Low

$\neg$ Intermediate

High

Low-censored

Intermediate-censored

High-censored

Figure 2 Kaplan-Meier analysis of EFS and PFS in chronic-phase chronic myeloid leukemia patients according to Sokal (low vs intermediate vs high), Hasford (low vs intermediate vs high), and EUTOS (low vs high) scores.

Notes: (A) Kaplan-Meier analysis showing the association of EFS with EUTOS (low vs high) scores (log-rank test, $P=0.289$ ), Sokal (low vs intermediate vs high) scores (logrank test, $P<0.00 \mathrm{I}$ ) and Hasford (low vs intermediate vs high) scores (log-rank test, $P=0.005$ ). (B) Kaplan-Meier analysis showing the association of PFS with EUTOS (low vs high) scores (log-rank test, $P=0.0 \mathrm{I}$ I), Sokal (low vs intermediate vs high) scores (log-rank test, $P=0.007$ ) and Hasford (low vs intermediate vs high) scores (log-rank test, $P<0.001$ ).

Abbreviations: EFS, event-free survival; EUTOS, European Treatment Outcome Study; PFS, progression-free survival.

Hasford score (HR=2.608, 95\% CI: 1.473-4.617, $P=0.001)$ were independently associated with $3 \mathrm{~m}-\mathrm{CCyR}$. Finally, age (HR=0.964, 95\% CI: 0.934-0.995, $P=0.023$ ), and proportion of blasts (HR=1.805, 95\% CI: $1.531-2.127, P<0.001$ ) was independently associated with $12 \mathrm{~m}-\mathrm{CCyR}$ (Table 2 ).

\section{Discussion}

Identifying the right scoring system for the prognosis of patients with CP-CML undergoing imatinib therapy is controversial. Therefore, the aim of the present study was to compare the capacity of three prognostic systems (Sokal, Hasford, and EUTOS) to predict outcome in 210 Chinese patients with CP-CML undergoing imatinib treatment.

Within the last decade, no epidemiological survey of CML was performed in Anhui Province, People's Republic of
China. In this study, the median age of CML onset was similar to that in People's Republic of China in the last century, ie, $40-50$ years, which is significantly lower than the onset age observed in western countries (60-65 years). ${ }^{1,5}$ The male-tofemale ratio was 1.5:1, similar to the ratio of 1.2:1 observed in other countries. ${ }^{25}$ The reason for the younger occurrence of CML in People's Republic of China is unknown.

According to the Sokal score, 28 (13.3\%) patients were high risk, 99 (47.1\%) were intermediate risk, and $83(39.5 \%)$ were low risk. According to the Hasford score, 17 (8.1\%) patients were high risk, 92 (43.8\%) were intermediate risk, and 101 (48.1\%) were low risk. According to the EUTOS score, $42(20.0 \%)$ patients were high risk and 168 (80.0\%) were low risk. Kaplan-Meier analyses were performed to assess the 3-month and 12-month CCyR, EFS, and PFS in each EUTOS, 
Table 2 Multivariate Cox regression analyses of clinicopathological variables and EFS, PFS, 3m-CCyR, and I2m-CCyR in patients with chronic-phase chronic myeloid leukemia

\begin{tabular}{|c|c|c|c|c|c|c|c|c|c|c|c|c|}
\hline \multirow[t]{2}{*}{ Variables } & \multicolumn{3}{|l|}{ EFS } & \multicolumn{3}{|l|}{ PFS } & \multicolumn{3}{|c|}{ 3m-CCyR } & \multicolumn{3}{|c|}{ I2m-CCyR } \\
\hline & HR & $95 \% \mathrm{Cl}$ & $P$ & HR & $95 \% \mathrm{Cl}$ & $P$ & HR & $95 \% \mathrm{Cl}$ & $P$ & HR & $95 \% \mathrm{Cl}$ & $\boldsymbol{P}$ \\
\hline Age & 0.949 & $0.919-0.980$ & 0.002 & 0.909 & $0.861-0.959$ & 0.001 & - & & & 0.964 & $0.934-0.995$ & 0.023 \\
\hline Proportion of blasts (\%) & 2.178 & $1.810-2.620$ & $<0.001$ & 2.966 & $2.057-4.277$ & $<0.001$ & 1.447 & $1.262-1.659$ & $<0.001$ & 1.805 & $1.531-2.127$ & $<0.001$ \\
\hline White blood cell count & - & & & 0.996 & $0.993-0.999$ & 0.020 & - & & & - & & \\
\hline Platelet count & 1.002 & $1.000-1.004$ & 0.045 & - & & & & & & & & \\
\hline Hasford & - & & & - & & & 2.608 & $1.473-4.617$ & 0.001 & - & & \\
\hline
\end{tabular}

Abbreviations: $\mathrm{Cl}$, confidence interval; EFS, event-free survival; HR, hazard ratio; PFS, progression-free survival; CCyR, complete cytogenetic response.

Sokal, and Hasford score category. The 3-month CCyR was lower in patients with high EUTOS scores, with intermediate or high Sokal scores, and with intermediate or high Hasford scores. The 12-month CCyR was lower in patients with high EUTOS scores, with intermediate or high Sokal scores, and with intermediate or high Hasford scores. EFS was lower with intermediate or high Sokal scores and with intermediate or high Hasford scores. There was no association between EFS and EUTOS scores; however, PSF was associated with EUTOS score. PFS was lower in patients with high EUTOS scores, with intermediate or high Sokal scores, and with intermediate or high Hasford scores. Multivariate Cox regression analyses revealed that only Hasford score were independently associated with 3-month CCyR.

Previously published reports of the EUTOS scoring system have mostly been conducted in Europe and were mostly single-center studies, in which the ratio of high-risk patients to the overall patient population was relatively low, ${ }^{16,18,20} \mathrm{ie}$, around $8 \%-25 \%$. In this study of a Chinese population, the proportion of high-risk patients was $20 \%$, within the previously reported range (higher than those observed by Hasford et $a \mathrm{l},{ }^{12}$ Marin et $\mathrm{al}^{19}$ and Jabbour et $\mathrm{al}{ }^{18}$ but lower than the one observed by Tao et $\mathrm{al}^{20}$ ). One report of EUTOS scoring in a Japanese population reported that $11 \%$ of patients were categorized as high risk, ${ }^{24}$ which was a lower level than in our study, but the Sokal and Hasford scores were similar to those previously reported in the Japanese study and many others. ${ }^{10,12,16,24,25}$

In this study, age was associated with EFS, PFS, and 12-month CCyR in patients with CP-CML receiving TKI, in contrast with the results of the GIMEMA CML trial. ${ }^{26}$ In the GIMEMA CML trial, and another study, older patients experienced more adverse events due to imatinib, but the long-term outcomes were similar to that of the younger patients. ${ }^{20,26}$ Age is a factor included in the Sokal score. ${ }^{10}$ Therefore, whether age is a prognostic factor in patients with CP-CML under TKI treatment still needs to be confirmed.

Cox multivariate analysis using forward selection indicated that only Hasford scores were independently associated with 3-month CCyR; age, proportion of blasts, and platelet count were independently associated with EFS; and age, proportion of blasts and white blood cell were independently associated with PFS. Eosinophil levels are not included in the calculation of Sokal or EUTOS scores. Although we measured the individual parameters used to calculate the Sokal and EUTOS scores, no molecular or genetic factors were considered. More baseline data, including molecular indicators, should be included to better describe CML prognosis. Sokal et $\mathrm{al}^{10}$ included the $\mathrm{Ph}$ chromosome in the baseline data, but ultimately did not include this parameter in the equation used to calculate the Sokal score. However, considering that the $\mathrm{Ph}$ chromosome was included in the measurement of the CCyR outcome, our results suggest that the Ph chromosome or the fusion gene might be a useful parameter of a revised score. However, further studies will be required to assess the value of any new score.

As reported previously by Höglund et $\mathrm{a}^{25}$ we observed statistically significant differences in the EFS of CP-CML patients in the Sokal high-risk group and the low- and intermediate-risk groups. PFS and CCyR were previously reported to be associated with the Sokal and EUTOS scores, as we also observed. ${ }^{20}$ Another study showed no association between Sokal score and EFS, while EFS was reported to be associated with Hasford and EUTOS scores. ${ }^{16}$ However, a recent study in a Japanese population reported that the EUTOS score was not associated with EFS, overall survival, or PFS. ${ }^{24}$ Our results suggest that even if all three scoring systems were associated with EFS, PFS, and 3-month and 12-month CCyR in Kaplan-Meier analyses (except EFS with EUTOS), only the Hasford score was independently associated with 3-month CCyR, while EUTOS score and Sokal score were not independently associated with any of these outcomes. Further studies will be required to assess the potential geographical and genetic differences between different populations of patients with CP-CML and to assess the prognostic value of these scores.

The present study suffers from some limitations. Few patients were distributed in the high-risk groups, and 
our conclusions need to be verified in larger samples. Additionally, we could not rule out the possibility that the data collected from a single-center study resulted in a relatively higher ratio due to a number of biases, such as referral, diagnosis, and observation biases. In addition, the EUTOS score system defined CCyR as $0 \%$ marrow cell metaphase after 18 months of therapy. As we reported only 3-month and 12-month CCyR, we cannot confirm that EUTOS better predicts 18-month CCyR. In addition, no death was observed in the present study. The evaluation of the molecular response was not part of the routine practice at our hospital during the study period. A longer term study reporting 18-month CCyR, overall survival, and 5-year disease-free survival may yield different results. This was a retrospective study with all the inherent biases and shortcomings, and a prospective trial should be performed. Accordingly, further prospective, larger, multicenter, longer studies are necessary to address these limitations and further probe the utility of EUTOS in this population.

\section{Acknowledgments}

This study was supported by the Natural Science Foundation of Science and Technology Agency of Anhui Province, People's Republic of China (grant no 11010402168).

\section{Author contributions}

All authors contributed toward data analysis, drafting and revising the paper and agree to be accountable for all aspects of the work.

\section{Disclosure}

The authors declare that they have no competing interest.

\section{References}

1. Baccarani M, Dreyling M; ESMO Guidelines Working Group. Chronic myeloid leukaemia: ESMO Clinical Practice Guidelines for diagnosis, treatment and follow-up. Ann Oncol. 2010;21(suppl 5):v165-v167.

2. Quintas-Cardama A, Cortes JE. Chronic myeloid leukemia: diagnosis and treatment. Mayo Clin Proc. 2006;81(7):973-988.

3. Hehlmann R, Hochhaus A, Baccarani M, European L. Chronic myeloid leukaemia. Lancet. 2007;370(9584):342-350.

4. Goldman JM, Melo JV. Chronic myeloid leukemia - advances in biology and new approaches to treatment. $N$ Engl J Med. 2003;349(15): 1451-1464.

5. Baccarani M, Cortes J, Pane F, et al; European LeukemiaNet. Chronic myeloid leukemia: an update of concepts and management recommendations of European LeukemiaNet. J Clin Oncol. 2009;27(35): 6041-6051.

6. Hughes TP, Hochhaus A, Branford S, et al; IRIS investigators. Longterm prognostic significance of early molecular response to imatinib in newly diagnosed chronic myeloid leukemia: an analysis from the International Randomized Study of Interferon and STI571 (IRIS). Blood. 2010;116(19):3758-3765.
7. Druker BJ, Tamura S, Buchdunger E, et al. Effects of a selective inhibitor of the Abl tyrosine kinase on the growth of Bcr-Abl positive cells. Nat Med. 1996;2(5):561-566.

8. Druker BJ, Guilhot F, O'Brien SG, et al; IRIS Investigators. Five-year follow-up of patients receiving imatinib for chronic myeloid leukemia. N Engl J Med. 2006;355(23):2408-2417.

9. de Lavallade H, Apperley JF, Khorashad JS, et al. Imatinib for newly diagnosed patients with chronic myeloid leukemia: incidence of sustained responses in an intention-to-treat analysis. J Clin Oncol. 2008; 26(20):3358-3363.

10. Sokal JE, Cox EB, Baccarani M, et al. Prognostic discrimination in "goodrisk" chronic granulocytic leukemia. Blood. 1984;63(4):789-799.

11. Hasford J, Pfirrmann M, Hehlmann R, et al. A new prognostic score for survival of patients with chronic myeloid leukemia treated with interferon alfa. Writing Committee for the Collaborative CML Prognostic Factors Project Group. J Natl Cancer Inst. 1998;90(11):850-858.

12. Hasford J, Baccarani M, Hoffmann V, et al. Predicting complete cytogenetic response and subsequent progression-free survival in 2,060 patients with CML on imatinib treatment: the EUTOS score. Blood. 2011;118(3):686-692.

13. XXXX. EUTOS for chronic myeloid leukemia shows improved management and dose optimization is required. Future Oncol. 2008;4(6):758.

14. Hoffmann V, Baccarani M, Hasford J, et al. The EUTOS CML score aims to support clinical decision-making. Blood. 2012;119(12):2966-2967.

15. Hoffmann VS, Baccarani M, Lindoerfer D, et al. The EUTOS prognostic score: review and validation in 1,288 patients with CML treated frontline with imatinib. Leukemia. 2013;27(10):2016-2022.

16. Uz B, Buyukasik Y, Atay H, et al. EUTOS CML prognostic scoring system predicts ELN-based 'event-free survival' better than Euro/ Hasford and Sokal systems in CML patients receiving front-line imatinib mesylate. Hematology. 2013;18(5):247-252.

17. Chinese Society of H. [Treatment guidelines of chronic myeloid leukemia]. Zhonghua Xue Ye Xue Za Zhi. 2011;32(6):426-432.

18. Jabbour E, Cortes J, Nazha A, et al. EUTOS score is not predictive for survival and outcome in patients with early chronic phase chronic myeloid leukemia treated with tyrosine kinase inhibitors: a single institution experience. Blood. 2012;119(19):4524-4526.

19. Marin D, Ibrahim AR, Goldman JM. European Treatment and Outcome Study (EUTOS) score for chronic myeloid leukemia still requires more confirmation. J Clin Oncol. 2011;29(29):3944-3945.

20. Tao Z, Liu B, Zhao Y, et al. EUTOS score predicts survival and cytogenetic response in patients with chronic phase chronic myeloid leukemia treated with first-line imatinib. Leuk Res. 2014;38(9):1030-1035.

21. Baccarani M, Deininger MW, Rosti G, et al. European LeukemiaNet recommendations for the management of chronic myeloid leukemia: 2013. Blood. 2013;122(6):872-884.

22. Kantarjian H, O'Brien S, Jabbour E, et al. Impact of treatment end point definitions on perceived differences in long-term outcome with tyrosine kinase inhibitor therapy in chronic myeloid leukemia. J Clin Oncol. 2011;29(23):3173-3178.

23. Zelenetz AD, Wierda WG, Abramson JS, etal. Non-Hodgkin's Lymphomas, version 3.2012. J Natl Compr Canc Netw. 2012;10(12):1487-1498.

24. Yamamoto E, Fujisawa S, Hagihara M, et al. European Treatment and Outcome Study score does not predict imatinib treatment response and outcome in chronic myeloid leukemia patients. Cancer Sci. 2014;105(1):105-109.

25. Höglund M, Sandin F, Hellström K, et al. Tyrosine kinase inhibitor usage, treatment outcome, and prognostic scores in CML: report from the population-based Swedish CML registry. Blood. 2013;122(7): 1284-1292.

26. Rosti G, Iacobucci I, Bassi S, et al. Impact of age on the outcome of patients with chronic myeloid leukemia in late chronic phase: results of a phase II study of the GIMEMA CML Working Party. Haematologica. 2007;92(1):101-105. 


\section{Publish your work in this journal}

OncoTargets and Therapy is an international, peer-reviewed, open access journal focusing on the pathological basis of all cancers, potential targets for therapy and treatment protocols employed to improve the management of cancer patients. The journal also focuses on the impact of management programs and new therapeutic agents and protocols on

patient perspectives such as quality of life, adherence and satisfaction. The manuscript management system is completely online and includes a very quick and fair peer-review system, which is all easy to use. Visit http://www.dovepress.com/testimonials.php to read real quotes from published authors.

Submit your manuscript here: http://www.dovepress.com/oncotargets-and-therapy-journal 